\title{
Responses of hadrons to chemical potential at finite temperature
}

\author{
S. Choe, ${ }^{1}$ Ph. de Forcrand, ${ }^{2,3}$ M. García Pérez, ${ }^{3}$ S. Hioki, ${ }^{4}$ Y. Liu,${ }^{1}$ H. Matsufuru,${ }^{5}$ \\ O. Miyamura, ${ }^{1}$ A. Nakamura, ${ }^{6}$ I.-O. Stamatescu, ${ }^{7,8}$ T. Takaishi, ${ }^{9}$ and T. Umeda ${ }^{10}$ \\ (QCD-TARO Collaboration) \\ ${ }^{1}$ Department of Physics, Hiroshima University, Higashi-Hiroshima 739-8526, Japan \\ ${ }^{2}$ Institut für Theoretische Physik, ETH-Hönggerberg, CH-8093 Zürich, Switzerland \\ ${ }^{3}$ Theory Division, CERN, CH-1211 Geneva 23, Switzerland \\ ${ }^{4}$ Department of Physics, Tezukayama University, Nara 631-8501, Japan \\ ${ }^{5}$ Yukawa Institute for Theoretical Physics, Kyoto University, Kyoto 606-8502, Japan \\ ${ }^{6}$ IMC, Hiroshima University, Higashi-Hiroshima 739-8521, Japan \\ ${ }^{7}$ Institut für Theoretische Physik, Universität Heidelberg, D-69120 Heidelberg, Germany \\ ${ }^{8}$ FEST, Schmeilweg 5, D-69118 Heidelberg, Germany \\ ${ }^{9}$ Hiroshima University of Economics, Hiroshima 731-0192, Japan \\ ${ }^{10}$ Center for Computational Physics, University of Tsukuba, Tsukuba 305-8577, Japan
}

(Dated: July 8, 2001, revised October 29, 2018)

\begin{abstract}
We present a framework to compute the responses of hadron masses to the chemical potential in lattice QCD simulations. As a first trial, the screening mass of the pseudoscalar meson and its first and second responses are evaluated. We present results on a $16 \times 8^{2} \times 4$ lattice with two flavors of staggered quarks below and above $T_{c}$. The responses to both the isoscalar and isovector chemical potentials are obtained. They show different behavior in the low and the high temperature phases, which may be explained as a consequence of chiral symmetry breaking and restoration, respectively.
\end{abstract}

PACS numbers: $12.38 . \mathrm{Gc}$

\section{INTRODUCTION}

It is well known that studying finite density QCD through lattice simulations is a very hard problem. The fermionic determinant at finite chemical potential is complex, and gives an oscillating behavior in quantum averages which makes simulations very inefficient. Since the naive quenched approximation at finite chemical potential leads to an essentially different world [1] , the use of dynamical fermions would be essential to extract the relevant physics. In spite of these difficulties, the study of hadrons in a finite baryonic environment is quite important [2, 3, 1], in view of recent experimental developments and of the theoretical interest in the phase structure of QCD. Search for the quark gluon plasma phase in high energy heavy ion collision experiments requires theoretical understanding of hadronic properties at finite temperature and density [5]. Moreover, some experimental results can be interpreted by assuming a shift in the mass and the width of the $\rho$ meson, induced by the dense nuclear medium even below the deconfinement transition [6].

In this paper we propose a new technique to investigate non-zero chemical potential using lattice QCD simulations. There are several approaches to circumvent the difficulty of studying a finite chemical potential system, and they seem successful to a limited extent [7, 8, 9]. In particular, the study of baryon number susceptibility at zero baryon density has reported an abrupt jump at the transition temperature 10]. There is in fact much interesting physical information which can be extracted from the behavior of a system at small chemical potential. Our strategy is to expand the hadronic quantities, such as masses, in the vicinity of zero chemical potential at finite temperature, and explore their changes through the response to the chemical potential at $\mu=0$. This allows to perform the numerical simulations with standard methods. The Taylor expansion in $\mu$ has also been used, and its properties discussed, in [9]. There, the behavior of observables, measured by standard methods as a function of an imaginary chemical potential $\mu$, is fitted by a Taylor series amenable to analytic continuation to real $\mu$ (assuming a large enough analyticity domain). Here we measure the Taylor coefficients directly, in a single simulation, at $\mu=0$, by calculating the derivatives of the relevant observables. Although the Taylor expansion cannot reproduce the non-analyticity inherent to a phase transition, it may suffice for observing the rounded, analytic behavior indicative of a phase transition in a finite volume, and for applying finite size scaling to probe the transition in the infinite volume limit. Our preliminary results have been reported in [11].

This paper is organized as follows. In the next section, we develop the basic formulae to evaluate the first and the second responses of hadron screening masses with respect to both of the isoscalar and the isovector chemical potentials. In Section III, we report on Monte Carlo simulations performed with two dynamical flavors of staggered quarks. We obtain the responses of the pseudoscalar meson mass, and discuss some implications of our results. Section [V] presents our conclusions. 


\section{CHEMICAL POTENTIAL RESPONSE OF HADRON MASSES AT FINITE TEMPERATURE}

This section develops the basic framework to observe the response of hadron masses with respect to the chemical potential. At fixed temperature $T$ and bare quark masses, the screening mass of a hadron is expanded in the form:

$$
\begin{aligned}
& \left.\frac{M(\mu)}{T}\right|_{\mu}=\left.\frac{M}{T}\right|_{\mu=0}+\left(\frac{\mu}{T}\right)\left(\frac{d M}{d \mu}\right)_{\mu=0} \\
& +\left(\frac{\mu}{T}\right)^{2} \frac{T}{2}\left(\frac{d^{2} M}{d \mu^{2}}\right)_{\mu=0}+O\left[\left(\frac{\mu}{T}\right)^{3}\right] .
\end{aligned}
$$

Assume that the spatial hadron correlator $C(x)$ is dominated by a single pole contribution,

$$
\begin{aligned}
C(x) & \equiv \sum_{y, z, t}\left\langle H(x, y, z, t) H(0,0,0,0)^{\dagger}\right\rangle \\
& =A\left(e^{-\hat{M} \hat{x}}+e^{-\hat{M}\left(L_{x}-\hat{x}\right)}\right),
\end{aligned}
$$

where $\hat{M}=a M$ and $\hat{x}=x / a . L_{x}$ is the lattice size in the $x$-direction. Generalization to a multi-pole situation is straightforward. In the case of a local hadron operator,
$A$ is represented as $A=\hat{\gamma} / 2 \hat{M}$ with the residue of the propagator, $\hat{\gamma}$. We employ a smeared hadron operator $H(x, y, z, t)$ to enhance the overlap with the ground state, and hence $A$ depends on the choice of sources. With a fixed type of smearing function, however, the behavior of $A$ as a function of the chemical potential provides information on the coupling to the medium.

We take the first and second derivatives of the hadron correlator with respect to $\hat{\mu} \equiv a \mu=\mu /\left(N_{t} T\right)$, where $\mu$ is the chemical potential. While we here write down expressions which depend on a single chemical potential, it is straightforward to generalize to the case involving two flavors corresponding to $u$ and $d$ quarks. Later we introduce the isovector and the isoscalar chemical potential by setting $\mu_{u}$ and $\mu_{d}$ appropriately. The first and the second derivatives of $C(x)$ with respect to the chemical potential lead to

$$
\begin{aligned}
& \frac{1}{C(x)} \frac{d C(x)}{d \hat{\mu}}=\frac{1}{A} \frac{d A}{d \hat{\mu}} \\
& +\frac{d \hat{M}}{d \hat{\mu}}\left\{\left(\hat{x}-\frac{L_{x}}{2}\right) \tanh \left[\hat{M}\left(\hat{x}-\frac{L_{x}}{2}\right)\right]-\frac{L_{x}}{2}\right\},
\end{aligned}
$$

and

$$
\begin{aligned}
\frac{1}{C(x)} \frac{d^{2} C(x)}{d \hat{\mu}^{2}}= & \frac{1}{A} \frac{d^{2} A}{d \hat{\mu}^{2}}+\left(\frac{2}{A} \frac{d A}{d \hat{\mu}} \frac{d \hat{M}}{d \hat{\mu}}+\frac{d^{2} \hat{M}}{d \hat{\mu}^{2}}\right)\left\{\left(\hat{x}-\frac{L_{x}}{2}\right) \tanh \left[\hat{M}\left(\hat{x}-\frac{L_{x}}{2}\right)\right]-\frac{L_{x}}{2}\right\} \\
& +\left(\frac{d \hat{M}}{d \hat{\mu}}\right)^{2}\left\{\left(\hat{x}-\frac{L_{x}}{2}\right)^{2}+\frac{L_{x}^{2}}{4}-L_{x}\left(\hat{x}-\frac{L_{x}}{2}\right) \tanh \left[\hat{M}\left(\hat{x}-\frac{L_{x}}{2}\right)\right]\right\} .
\end{aligned}
$$

$C(x)$ and the derivatives of $C(x)$ are calculated from lattice simulations. Then, using the right-hand side of Eqs. (3) and (4), the first and the second responses of hadron masses and couplings are determined.

The next step is to relate the derivatives of the correlator to quantities which are measured in the lattice simulations. In this work, we consider the flavor nonsinglet mesons in QCD with two flavors. The hadron correlator is then given by

$$
\left\langle H(n) H(0)^{\dagger}\right\rangle=\langle G\rangle,
$$

where $G$ is the meson propagator

$$
G=\operatorname{Tr}\left[P\left(\hat{\mu}_{u}\right)_{n 0} \Gamma P\left(\hat{\mu}_{d}\right)_{0 n} \Gamma^{\dagger}\right] .
$$

Here $P(\hat{\mu})$ is the quark propagator at finite chemical potential, and $\Gamma$ is the Dirac matrix which specifies the spin of the meson. The quark propagator is related to the Dirac operator $D[U ; \hat{\mu}]$ in the background gauge field configuration $U$ as

$$
P(\hat{\mu})=D[U ; \hat{\mu}]^{-1} .
$$

The expectation value of operator $O,\langle O\rangle$, stands for

$$
\langle O\rangle=\frac{\int[d U] O \Delta e^{-S_{G}}}{\int[d U] \Delta e^{-S_{G}}},
$$

where $S_{G}$ is the gluonic action and $\Delta$ is the fermion determinant,

$$
\Delta=\operatorname{det} D\left[U ; \hat{\mu}_{u}\right] \operatorname{det} D\left[U ; \hat{\mu}_{d}\right] .
$$

Then, the first and the second derivatives are represented as

$$
\begin{gathered}
\frac{d}{d \hat{\mu}}\left\langle H(n) H(0)^{\dagger}\right\rangle=\left\langle\dot{G}+G \frac{\dot{\Delta}}{\Delta}\right\rangle-\langle G\rangle\left\langle\frac{\dot{\Delta}}{\Delta}\right\rangle, \\
\frac{d^{2}}{d \hat{\mu}^{2}}\left\langle H(n) H(0)^{\dagger}\right\rangle=\left\langle\ddot{G}+2 \dot{G} \frac{\dot{\Delta}}{\Delta}+G \frac{\ddot{\Delta}}{\Delta}\right\rangle \\
-2\left\langle\dot{G}+G \frac{\dot{\Delta}}{\Delta}\right\rangle\left\langle\frac{\dot{\Delta}}{\Delta}\right\rangle
\end{gathered}
$$




$$
-\langle G\rangle\left\{\left\langle\frac{\ddot{\Delta}}{\Delta}\right\rangle-2\left\langle\frac{\dot{\Delta}}{\Delta}\right\rangle^{2}\right\},
$$

where the dotted $\dot{O}$ and $\ddot{O}$ denote the first and the second derivatives of the operator $O$ with respect to $\hat{\mu}$.

At zero chemical potential, we have simpler expressions, since

$$
\left\langle\frac{\dot{\Delta}}{\Delta}\right\rangle=0 \quad \text { for } \quad \hat{\mu}=0 .
$$

Eq. (12) corresponds to the fact that the average baryon number density vanishes at $\hat{\mu}=0$. Actually, we see that $d(\operatorname{det} D) / d \hat{\mu}=\operatorname{Tr}\left[\dot{D} D^{-1}\right] \operatorname{det} D$ is anti-hermitian at $\hat{\mu}=$ 0 :

$$
\begin{aligned}
\operatorname{Tr}\left[\dot{D} D^{-1}\right] & =\operatorname{Tr}\left[\dot{D} \gamma_{5} \gamma_{5} D^{-1}\right] \\
& =\operatorname{Tr}\left[\left(-\gamma_{5} \dot{D}^{\dagger}\right)\left(D^{\dagger}\right)^{-1} \gamma_{5}\right] \\
& =-\operatorname{Tr}\left[\dot{D} D^{-1}\right]^{*}
\end{aligned}
$$

This means that $d(\operatorname{det} D) / d \hat{\mu}$ changes sign under the transformation $U \rightarrow U^{\dagger}$. Since the measure and the gluonic action are invariant under this transformation, its expectation value vanishes 12]. Thus, at zero chemical potential, Eqs. (10) and (11) turn into

$$
\begin{aligned}
\frac{d}{d \hat{\mu}}\left\langle H(n) H(0)^{\dagger}\right\rangle= & \left\langle\dot{G}+G \frac{\dot{\Delta}}{\Delta}\right\rangle, \\
\frac{d^{2}}{d \hat{\mu}^{2}}\left\langle H(n) H(0)^{\dagger}\right\rangle= & \left\langle\ddot{G}+2 \dot{G} \frac{\dot{\Delta}}{\Delta}+G \frac{\ddot{\Delta}}{\Delta}\right\rangle \\
& -\langle G\rangle\left\langle\frac{\ddot{\Delta}}{\Delta}\right\rangle .
\end{aligned}
$$

We investigate derivatives with respect to both the isoscalar and the isovector types of chemical potential. The isoscalar chemical potential is conjugate to the total quark density. In this paper we study the response to the isoscalar chemical potential by setting

$$
\hat{\mu}_{S}=\hat{\mu}_{u}=\hat{\mu}_{d},
$$

and for the isovector case

$$
\hat{\mu}_{V}=\hat{\mu}_{u}=-\hat{\mu}_{d} .
$$

Note that Son and Stephanov proposed a model corresponding to the isovector case as a good test bed for chemical potential effects in QCD 13. An advantage of setting $\hat{\mu}_{u}=-\hat{\mu}_{d}$ is that the fermion determinant is positive, and therefore the problem becomes tractable with standard lattice techniques. Here we do not make use of this advantage and still study the dependence on $\hat{\mu}_{V}$ by performing a Taylor expansion around $\hat{\mu}_{V}=0$. Future simulations at non-zero $\hat{\mu}_{V}$ would also constitute a good test of the performance of our approach.

Our simulations are performed with $N_{f}=2$ dynamical quarks of the staggered fermion type. The fermion operator and its derivatives are

$$
\begin{aligned}
& D[U ; \hat{\mu}]_{n, m}=m a \delta_{n, m} \\
& \quad+\frac{1}{2} \sum_{\sigma=x, y, z} \eta_{\sigma}(n)\left[U_{\sigma}(n) \delta_{n+\hat{\sigma}, m}-U_{\sigma}^{\dagger}(n-\hat{\sigma}) \delta_{n-\hat{\sigma}, m}\right] \\
& \quad+\frac{1}{2} \eta_{t}(n)\left[U_{t}(n) e^{\hat{\mu}} \delta_{n+\hat{t}, m}-U_{t}^{\dagger}(n-\hat{t}) e^{-\hat{\mu}} \delta_{n-\hat{t}, m}\right], \\
& \frac{d D}{d \hat{\mu}}=\frac{\eta_{t}(n)}{2}\left[U_{t}(n) e^{\hat{\mu}} \delta_{n+\hat{t}, m}+U_{t}^{\dagger}(n-\hat{t}) e^{-\hat{\mu}} \delta_{n-\hat{t}, m}\right]
\end{aligned}
$$

and

$\frac{d^{2} D}{d \hat{\mu}^{2}}=\frac{\eta_{t}(n)}{2}\left[U_{t}(n) e^{\hat{\mu}} \delta_{n+\hat{t}, m}-U_{t}^{\dagger}(n-\hat{t}) e^{-\hat{\mu}} \delta_{n-\hat{t}, m}\right]$,

where $\hat{\sigma}$ and $\hat{t}$ are unit vectors pointing along spatial and temporal directions, respectively.

Taking into account the four-fold degeneracy of the staggered fermion operator, the determinant factor $\Delta$ of $N_{f}=2$ fermions is then

$$
\Delta=\exp \left(\frac{1}{4} \operatorname{Tr} \ln D\left[U ; \hat{\mu}_{u}\right]+\frac{1}{4} \operatorname{Tr} \ln D\left[U ; \hat{\mu}_{d}\right]\right) .
$$

Explicit formulae for the isoscalar and the isovector cases are presented in Appendices A and B respectively. Appendix $\mathrm{C}$ gives the specific forms for staggered quarks.

\section{NUMERICAL RESULTS}

\section{A. Lattice setup and meson screening masses}

In this section, we describe the results of Monte Carlo (MC) simulations for the response of meson screening masses with respect to the chemical potentials below and above the chiral phase transition. Numerical simulations are performed on lattices of size $16 \times 8^{2} \times 4$ with the standard Wilson gauge action and with two dynamical flavors of staggered quarks. The configurations are generated with the R-algorithm with molecular dynamics step $\delta=0.01$ and trajectory length of 50 steps. For $N_{t}=4$, the critical coupling $\beta_{c}$ of the finite temperature phase transition has been determined as $\beta_{c}=5.271$ for $m a=0.0125$ and $\beta_{c}=5.288$ for $m a=0.025$, respectively [14, 15]. We perform numerical simulations at $m a=0.0125,0.017$ and 0.025 . The values of $\beta$ are listed in Table together with the number of configurations and rough estimates of the corresponding temperatures. To evaluate the traces of the various fermionic operators, the $Z_{2}$ noise method [16] is used, with 200 noise vectors. The real part of the average Polyakov loop, as shown in Figure 1, exhibits a rapid increase between about $\beta=5.27$ and 5.28 , as expected. 
TABLE I: Parameters of the simulations. $N_{\text {conf }}$ denotes the number of configurations on which the correlators are measured.

\begin{tabular}{cccc}
\hline \hline$m a$ & $\beta$ & $N_{\text {conf }}$ & $T / T_{C}$ \\
\hline 0.0125 & 5.26 & 600 & 0.99 \\
& 5.34 & 300 & 1.10 \\
\hline 0.0170 & 5.26 & 600 & 0.98 \\
& 5.34 & 300 & 1.09 \\
\hline 0.0250 & 5.20 & 600 & 0.89 \\
& 5.26 & 600 & 0.96 \\
& 5.32 & 300 & 1.05 \\
& 5.34 & 300 & 1.07 \\
\hline \hline
\end{tabular}

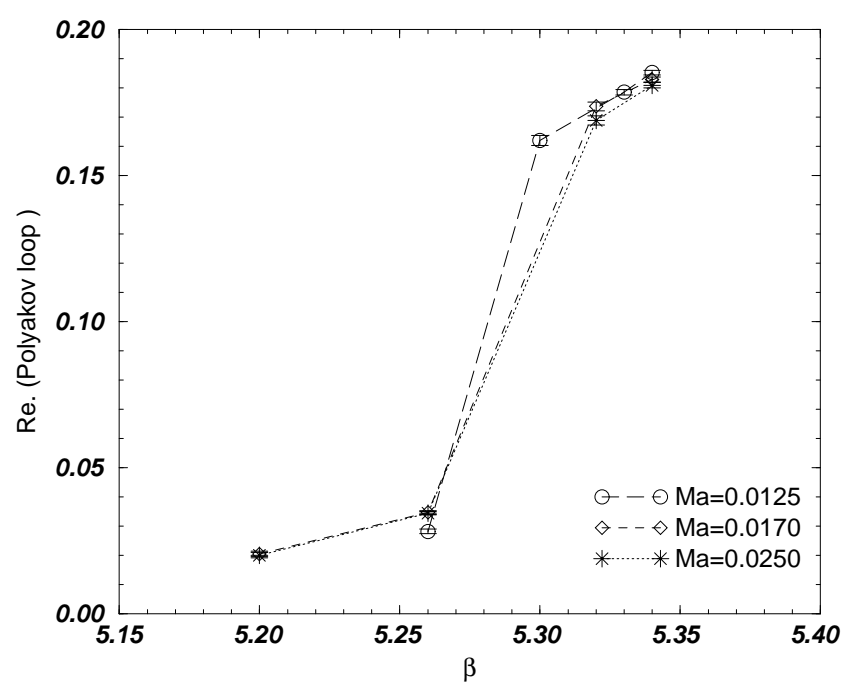

FIG. 1: Average value of the real part of Polyakov loop as a function of $\beta$.

Meson correlators are measured on configurations separated by 20 trajectories. We use a corner-type wall source [17] after Coulomb gauge fixing in each $(y, z, t)$ hyperplane. We determine the meson screening masses $M$ by fitting the $\mathrm{MC}$ data to the single exponential form, Eq. (2), with the fitting range $x=1-15$. As shown in Figure 2, the fit is successfully applied for the pseudoscalar(PS) meson channel. The extracted PS meson screening mass is listed in Table II. Below $T_{c}$, the present lattice extent in the shorter spatial directions is not sufficiently large to extract reliably the PS meson mass. Since it is known that the finite size effects on hadron masses are rather severe with dynamical staggered fermions [18, 19, , one should use lattices with $L \cdot m_{\pi}$ larger than $\sim 5$ to avoid finite size effects, and hence larger lattice sizes are obviously required for a quantitative analysis. Goals of the present study are, however, to show the applicability of the method to extract the responses of meson mass to the chemical potentials, and to observe the qualitatively different behavior below and above $T_{c}$ giving us hints for future detailed studies.
TABLE II: Responses of the PS meson to the isoscalar chemical potential $\hat{\mu}_{S}$.

\begin{tabular}{cccccc}
\hline \hline$m a$ & $\beta$ & $\hat{M}$ & $\frac{1}{A} \frac{d^{2} A}{d \hat{\mu}^{2}}$ & $\frac{d^{2} \hat{M}}{d \hat{\mu}^{2}}$ & $\frac{1}{\hat{\gamma}} \frac{d^{2} \hat{\gamma}}{d \hat{\mu}^{2}}$ \\
\hline 0.0125 & 5.26 & $0.2956(2)$ & $-1.4(20)$ & $0.17(35)$ & $-0.8(23)$ \\
& 5.34 & $0.7513(11)$ & $-4.23(49)$ & $5.39(10)$ & $2.95(51)$ \\
\hline 0.0170 & 5.26 & $0.3506(2)$ & $-1.5(14)$ & $0.30(26)$ & $-0.6(16)$ \\
& 5.34 & $0.7421(35)$ & $-3.68(75)$ & $5.82(16)$ & $4.16(78)$ \\
\hline 0.0250 & 5.20 & $0.4061(2)$ & $-0.4(14)$ & $0.16(26)$ & $0.0(16)$ \\
& 5.26 & $0.4218(2)$ & $-0.8(11)$ & $0.29(20)$ & $-0.1(12)$ \\
& 5.32 & $0.6926(11)$ & $-4.65(91)$ & $5.17(20)$ & $2.82(96)$ \\
& 5.34 & $0.7534(7)$ & $-3.17(41)$ & $4.43(8)$ & $2.71(42)$ \\
\hline \hline
\end{tabular}

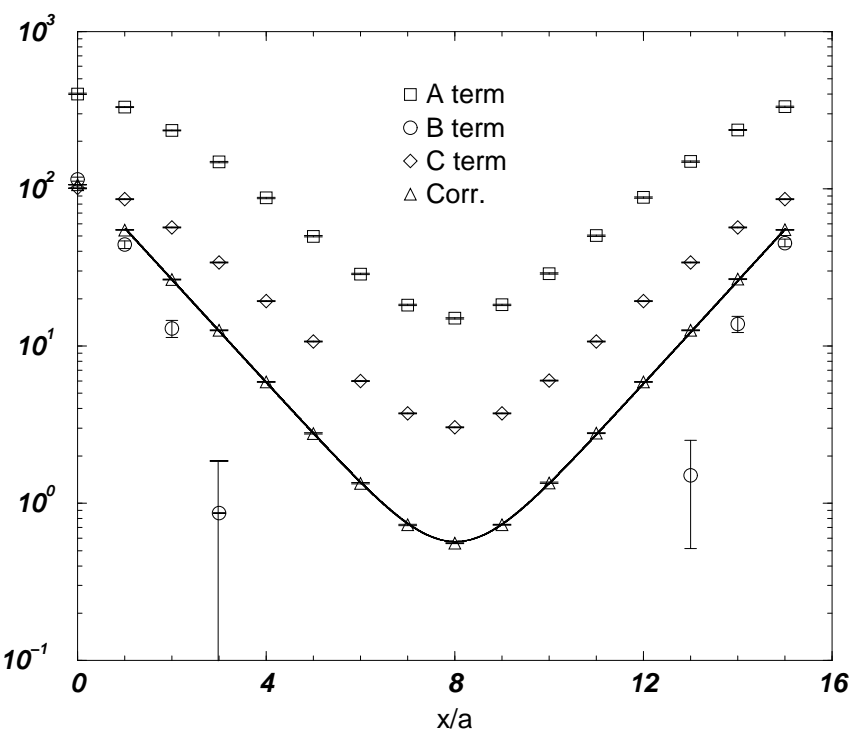

FIG. 2: Pseudoscalar correlator and several contributions to its responses to $\hat{\mu}_{S, V}$ (see Eq. (22)) at $\beta=5.34$ and $m a=$ 0.025. The correlator is fitted to the single pole form, Eq. (2).

Therefore we present also the results below $T_{c}$ in spite of the large systematic uncertainty. The screening mass of the PS meson at $m a=0.025$ as a function of $T / T_{c}$ is shown in Figure 3 .

In the case of the vector meson correlator, although the procedure is in principle also applicable, the statistical fluctuations prevent us from reaching any relevant conclusion at this stage, and therefore we do not report results for the vector channel in the following.

\section{B. Responses of the PS meson mass to the isoscalar chemical potential}

Several terms contribute to the derivatives of the meson correlator with respect to the chemical potential: see Eqs. (B5), (C3), and (C4) in Appendices. As represen- 


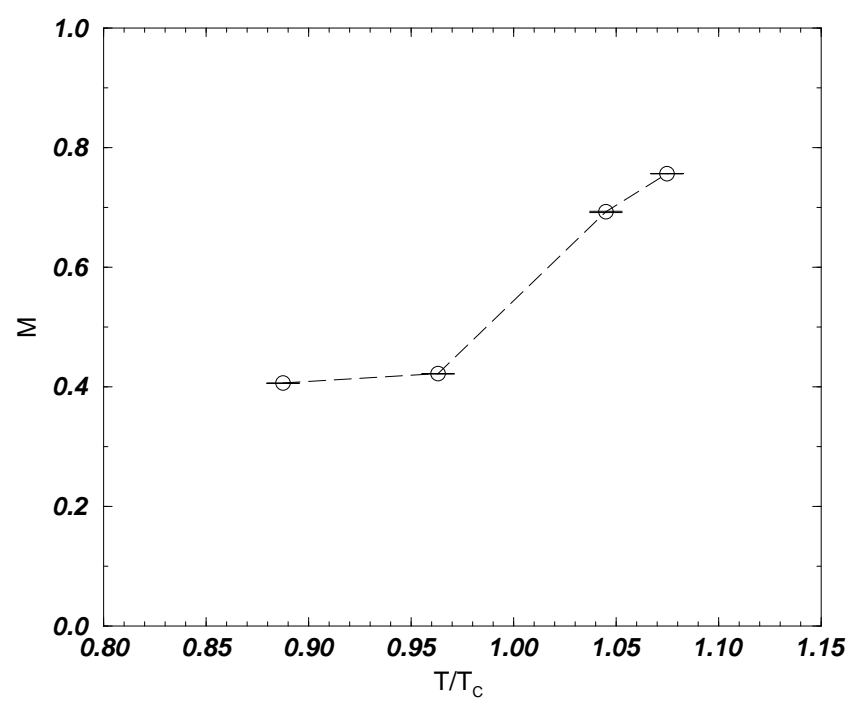

FIG. 3: Screening mass of the PS meson in lattice units at $m a=0.025 . \beta_{C}$ is taken from [15]. Values of $T / T_{C}$ are estimated by using the two-loop $\beta$-function.

tative terms, in Figure 2 we show

$$
\begin{array}{ll}
(A): & 2 \sum_{y, z, t} \operatorname{Re}\left\langle\operatorname{Tr}\left[(P \dot{D} P)_{n 0}(P \dot{D} P)_{n 0}^{\dagger}\right]\right\rangle, \\
(B): \quad & 4 \sum_{y, z, t} \operatorname{Re}\left\langle\operatorname{Tr}\left[(P \dot{D} P \dot{D} P)_{n 0} P_{n 0}^{\dagger}\right]\right\rangle, \\
(C): & 2 \sum_{y, z, t} \operatorname{Re}\left\langle\operatorname{Tr}\left[(P \ddot{D} P)_{n 0} P_{n 0}^{\dagger}\right]\right\rangle .
\end{array}
$$

All of them are determined with reasonable accuracy.

Let us start with the first response of the PS correlator to the chemical potential. Note that the first derivative with respect to the isoscalar chemical potential is identically zero (see Eq. (A10) in Appendix A). For the isovector chemical potential, Figure 1 shows $C^{-1} d C / d \hat{\mu}_{V}$ at $\beta=5.26$ and 5.34. At both temperatures, the values are very small. This is consistent with a comparative study in the Nambu-Jona-Lasinio model, which also predicts very small responses around the critical temperature [21].

We then consider the second responses. Figure 5 shows $C^{-1} d^{2} C / d \hat{\mu}^{2}$ at $\beta=5.26$ (below $T_{c}$ ) and 5.34 (above $T_{c}$ ) for the isoscalar and isovector chemical potentials. The solid curves represent the fits to Eq. (4), after fitting $C(x)$ to Eq. (2).

We determine the responses of the meson mass with respect to the chemical potentials as follows. As described in the last subsection, the meson correlator is first fitted to Eq. (2) to extract the screening mass, $\hat{M}$. Then, we fit the MC results for the derivatives of the meson correlator to Eqs. (3) and (4), substituting the determined value of $\hat{M}$. The derivatives of mass and coupling are then obtained as fitting parameters. Note that for $\hat{\mu}_{S}$ we omit the fitting step to Eq. (3), since the first response is strictly zero.
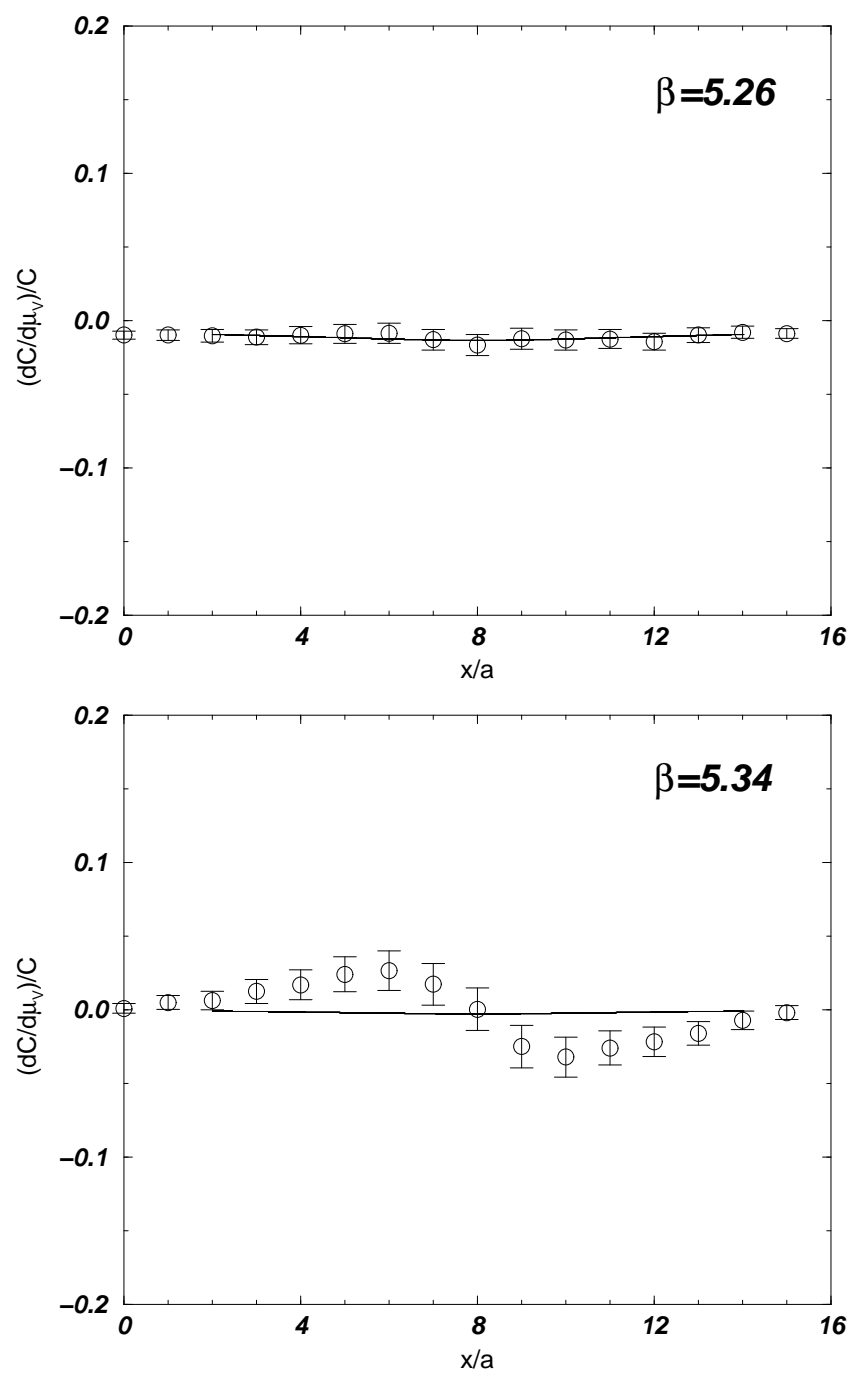

FIG. 4: The first response of the PS meson correlator at $\beta=5.26\left(T<T_{c}\right.$, top figure) and $\beta=5.34\left(T>T_{c}\right.$, bottom $)$. The quark mass is $m a=0.025$.

Results of the PS meson response to the isoscalar chemical potential are summarized in Table II. In the low temperature phase, the dependence of the mass on $\hat{\mu}_{S}$ is small. This behavior is to be expected, since, below the critical temperature and in the vicinity of zero $\hat{\mu}_{S}$, the PS meson is still a Goldstone boson. In fact, the extrapolated value to the chiral limit of the isoscalar response is consistent with zero, as shown in Figure 6. This is in contrast with the behavior above $T_{c}$, where $d^{2} \hat{M}^{2} / d \hat{\mu}^{2}$ seems to remain finite even in the chiral limit. In addition, our results suggest that the response of the coupling is small below $T_{c}$.

Above $T_{c}$, we first note that the correlator and its response are still well fitted by the single pole formulae, Eqs. (24). The screening masses are manifestly larger than those below $T_{c}$. This is consistent with previous work [10]. As pointed out above, the response of the mass above $T_{c}$ becomes large, as a reflection of the fact 

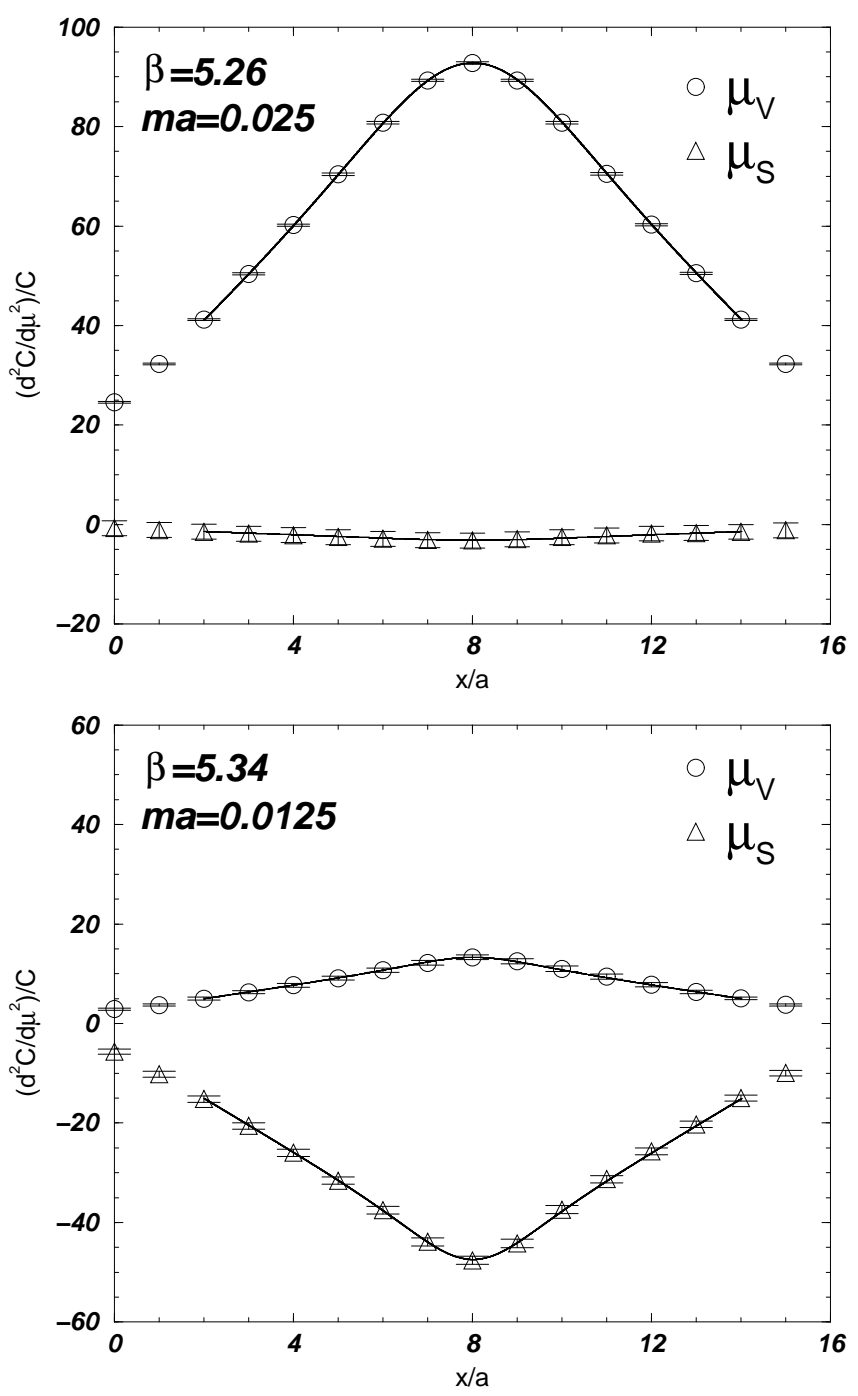

FIG. 5: The second responses of the PS meson correlator at $\beta=5.26$ and $m a=0.025$ ( $T<T_{c}$, top), and at $\beta=5.34$ and $m a=0.0125\left(T>T_{c}\right.$, bottom). The curves are fits to Eq. (4).

that the pion is no longer a Goldstone boson and as an indication of chiral symmetry restoration. We also find that the response of the coupling $\hat{\gamma}$ increases above $T_{c}$, as shown in Figure 8, which may indicate a larger overlap of the wall-source operator with free quarks in the plasma phase. Note that $\left(d^{2} \hat{\gamma} / d \hat{\mu}^{2}\right) / \hat{\gamma}$ does not depend on the choice of the source normalization.

\section{Responses of the PS meson mass to the isovector chemical potential}

Results for the isovector chemical potential are summarized in Table III. In the presence of the isovector chemical potential, $\pi^{+}$and $\pi^{-}$may have different masses. Here we consider the $\pi^{+}(u \bar{d})$ meson as shown in Eq. (6).
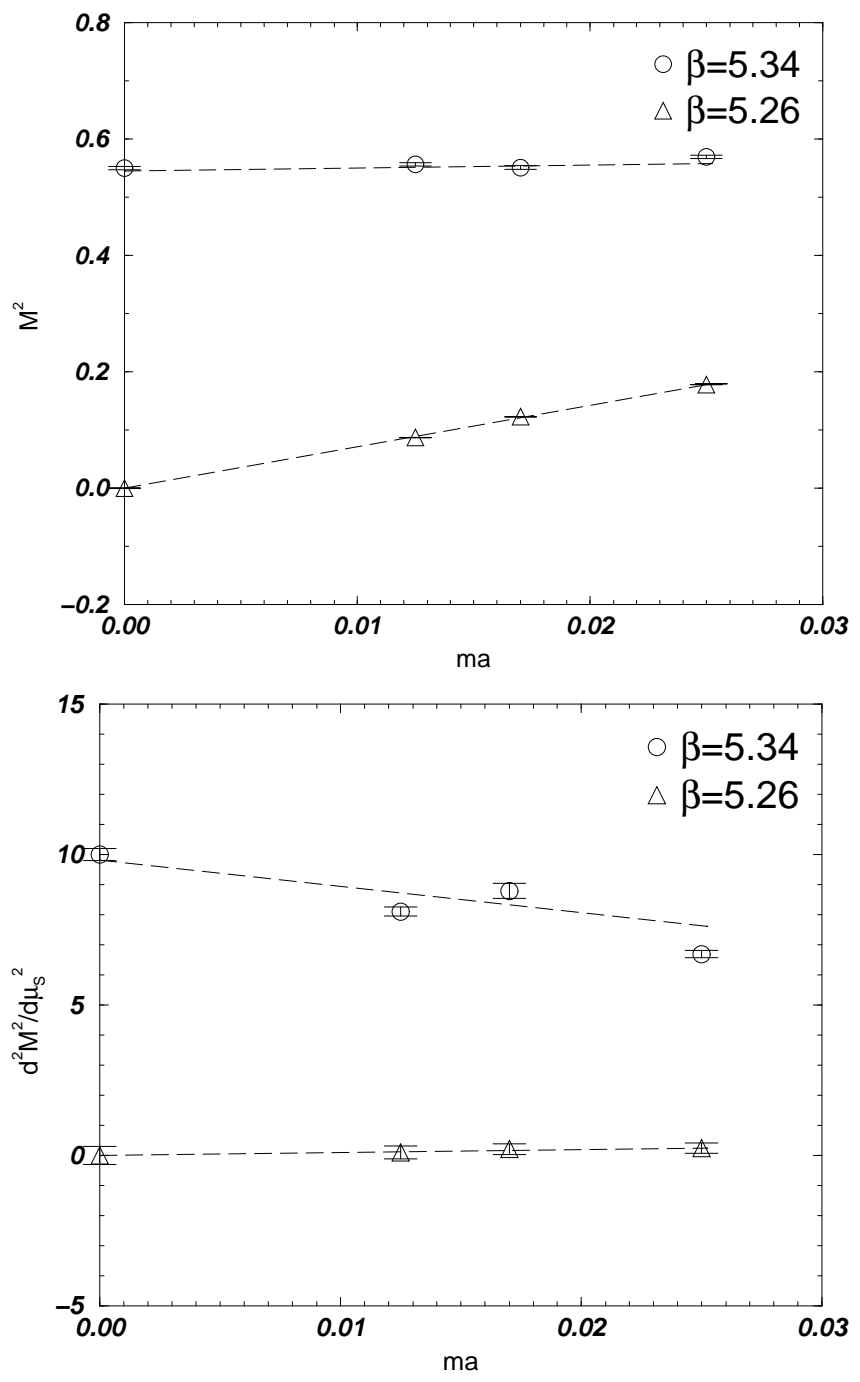

FIG. 6: $\hat{M}^{2}$ (top) and $d^{2} \hat{M}^{2} / d \hat{\mu}_{S}^{2}$ (bottom) for the PS meson versus $m a$. $\beta$ is 5.26 (triangles) and 5.34 (circles). Extrapolation to $m a=0$ is also shown.

In contrast to the case of the isoscalar chemical potential, the second order response of the mass is significantly large in the low temperature phase, and decreases in magnitude above $T_{c}$. The difference between the isoscalar and isovector chemical potentials is illustrated in Figure 7 . The response of $\hat{\gamma}$ also shows different behaviors in the confined and deconfined phases, illustrated in Figure 8.

These features are manifest even for a small quark mass parameter. Note that the isovector potential explicitly breaks the $u$ - $d$ symmetry, even if the two quarks have equal masses. The phase structure in the $\left(T,\left|\mu_{V}\right|\right)$ plane has been studied by Son and Stephanov 13. The original $S U(2)_{L+R}$ symmetry at non-zero quark mass and zero chemical potential is broken down to $U(1)_{L+R}$. At zero $T$ and for $\left|\mu_{V}\right|$ larger than the mass of the pseudoscalar, the system is in a different phase than at $\mu_{V}=0$. The ground state is a pion condensate and there is one massless Goldstone boson associated with the spontaneous 


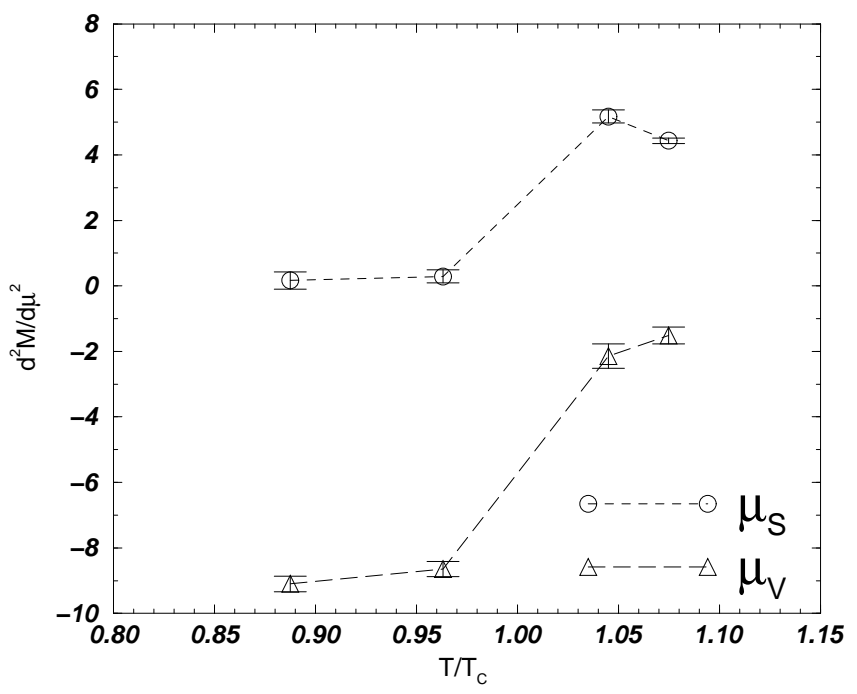

FIG. 7: Second responses $d^{2} \hat{M} / d \hat{\mu}_{S}^{2}$ and $d^{2} \hat{M} / d \hat{\mu}_{V}^{2}$ of the PS meson mass at $m a=0.025 . T / T_{c}$ is estimated as in Figure 3 .

breaking of the $U(1)_{L+R}$ symmetry. For $\left|\mu_{V}\right|=m_{P S}$, the critical temperature is $T=0$. At sufficiently high temperature, the condensate melts and the symmetry is restored. Due to the presence of the phase boundary, we do not expect to be able to reach the condensed phase by Taylor expanding around $\mu_{V}=0$. We can, however, hope to get some hints about the presence of the phase boundary while keeping $\left|\mu_{V}\right|<m_{P S}$. In this case, the system is in the same ground state as for zero chemical potential, there are no exact Goldstone modes and the three pions are massive.

An interesting point in this respect is that the second derivative of the mass is negative in the low temperature phase, in marked contrast with what happened for the isoscalar potential. The mass tends to decrease under the influence of the isovector chemical potential, reflecting the fact that for low temperature and chemical potential above the pion mass, a Goldstone mode appears [1, 13. This is more clearly shown by an expansion as in Eq. (11). At $\beta=5.26$ and $m a=0.017$, the data suggest

$$
\begin{aligned}
\left.\frac{M\left(\mu_{V}\right)}{T}\right|_{\mu_{V}} & =(1.4024 \pm 0.0008) \\
+ & (-0.0005 \pm 0.0010)\left(\frac{\mu_{V}}{T}\right) \\
& -(1.31 \pm 0.04)\left(\frac{\mu_{V}}{T}\right)^{2}+O\left[\left(\frac{\mu_{V}}{T}\right)^{3}\right] .
\end{aligned}
$$

The coefficient of the linear term is consistent with zero. Notice also in Table III that the lighter the quark mass, the stronger the response, a possible indication that for lighter pions the phase boundary is closer to the zero chemical potential axis, as suggested in 13.

In the high temperature phase, the dependence of the masses on $\mu_{V}$ decreases. Since the PS meson becomes heavier, the phase boundary to the pion condensate phase is farther away from the $\mu_{V}=0$ axis. The

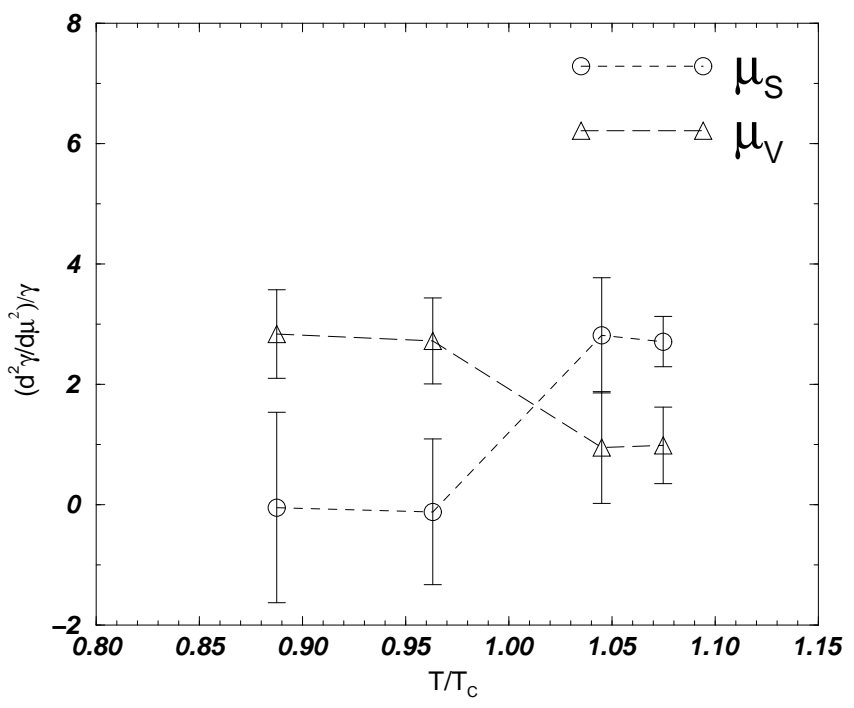

FIG. 8: Second responses of the couplings, $\hat{\gamma}^{-1} d^{2} \hat{\gamma} / d \hat{\mu}_{S}^{2}$, $\hat{\gamma}^{-1} d^{2} \hat{\gamma} / d \hat{\mu}_{V}^{2}$, for the PS meson at $m a=0.025$. $T / T_{c}$ is estimated as in Figure 3 .

weaker responses may be understood from this point of view.

\section{CONCLUSIONS}

In this work, we have developed a framework to study the response of hadrons to the chemical potential. It is based on Taylor expanding hadronic quantities about $\mu=0$. We show the first results of the first and second derivatives of the PS meson screening mass with respect to $\mu$. As shown in the previous section, the second order responses are sizable and reveal several characteristic features. The behavior of the responses for the PS meson seems to have close contact to the chiral symmetry restoration. For the isoscalar chemical potential $\mu_{S}$, the dependence of the PS mass on $\mu_{S}$ in the chiral limit is consistent with zero, reflecting the fact that at low temperature and small $\mu_{S}$ the pion is still a Goldstone boson. For the isovector chemical potential, we show features consistent with the phase structure proposed by Son and Stephanov [13]. The $u \bar{d}$ PS meson mass tends to decrease as a function of $\mu_{V}$ at a much stronger rate in the low temperature phase.

It is notable that a single hadron pole gives a good description for the response as well as for the correlator even at $\beta=5.34\left(T / T_{c} \approx 1.1\right)$ in the PS channel.

Since the present study is a first trial, our simulations have been performed on a rather small lattice. Since the difference between the dynamics of $N_{t}=4$ and $N_{t}=6$ lattices is sizable 20] and since the spatial volume should be large enough to accommodate the hadrons, further investigations on larger lattices are indispensable. The chemical potential responses of other quantities, such as the nucleon mass and the quark condensate, are impor- 
TABLE III: Responses of the PS meson correlator to the isovector chemical potential $\hat{\mu}_{V}$.

\begin{tabular}{|c|c|c|c|c|c|c|}
\hline$m a$ & $\beta$ & $\frac{1}{A} \frac{d A}{d \hat{\mu}}$ & $\frac{d \hat{M}}{d \hat{\mu}}$ & $\frac{1}{A} \frac{d^{2} A}{d \hat{\mu}^{2}}$ & $\begin{array}{l}\frac{d^{2} \hat{M}}{d \hat{\mu}^{2}} \\
\end{array}$ & $\frac{1}{\hat{\gamma}} \frac{d^{2} \hat{\gamma}}{d \hat{\mu}^{2}}$ \\
\hline \multirow[t]{2}{*}{0.0125} & 5.26 & $0.0029(57)$ & $-0.0001(12)$ & $47.46(71)$ & $-12.93(43)$ & $3.7(16)$ \\
\hline & 5.34 & $0.0047(93)$ & $0.0006(21)$ & $2.32(64)$ & $-1.32(32)$ & $0.56(77)$ \\
\hline \multirow[t]{2}{*}{0.0170} & 5.26 & $-0.0081(48)$ & $-0.0005(10)$ & $33.52(61)$ & $-10.49(33)$ & $3.6(11)$ \\
\hline & 5.34 & $0.0000(82)$ & $-0.0012(19)$ & $2.74(64)$ & $-1.48(32)$ & $0.75(78)$ \\
\hline \multirow[t]{4}{*}{0.0250} & 5.20 & $0.0062(39)$ & $0.0016(8)$ & $25.24(46)$ & $-9.10(23)$ & $2.84(74)$ \\
\hline & 5.26 & $-0.0080(37)$ & $0.0007(8)$ & $23.22(46)$ & $-8.64(23)$ & $2.72(71)$ \\
\hline & 5.32 & $-0.0054(64)$ & $-0.0020(14)$ & $4.04(75)$ & $-2.14(38)$ & $0.95(93)$ \\
\hline & 5.34 & $0.000(6)$ & $0.000(1)$ & $2.99(53)$ & $-1.51(26)$ & $0.99(60)$ \\
\hline
\end{tabular}

tant issues. Exploratory studies on these subjects are in progress.

\section{Acknowledgments}

This work is supported by Grant-in-Aide for Scientific Research by Monbusho, Japan (No.11694085, No.11740159, and No. 12554008). Simulations were performed on the HITACHI SR8000 at IMC, Hiroshima University. H. M. is supported by the Japan Society for the Promotion of Science for Young Scientists.

\section{APPENDIX A: FORMULAE FOR THE ISOSCALAR CHEMICAL POTENTIAL RESPONSE}

The quark propagator $P$ satisfies the relation

$$
P\left(\hat{\mu}_{d}\right)_{0 n}=\gamma_{5} P^{\dagger}\left(-\hat{\mu}_{d}\right)_{n 0} \gamma_{5}
$$

so that the meson correlator $G$ is given by

$$
G=\operatorname{Tr}\left[P\left(\hat{\mu}_{S}\right)_{n 0} \Gamma \gamma_{5} P\left(-\hat{\mu}_{S}\right)_{n 0}^{\dagger} \gamma_{5} \Gamma^{\dagger}\right]
$$

where "Tr" means the trace over spinor and color indices. Each propagator is expanded as

$$
\begin{aligned}
P(\hat{\mu})= & P-\hat{\mu} P \dot{D} P+\frac{\hat{\mu}^{2}}{2}(2 P \dot{D} P \dot{D} P-P \ddot{D} P) \\
& +O\left(\hat{\mu}^{3}\right), \\
P(-\hat{\mu})= & P+\hat{\mu} P \dot{D} P+\frac{\hat{\mu}^{2}}{2}(2 P \dot{D} P \dot{D} P-P \ddot{D} P) \\
& +O\left(\hat{\mu}^{3}\right),
\end{aligned}
$$

where $P$ and $D$ are the quark propagator and the Dirac operator at zero chemical potential, respectively, and the relation

$$
\dot{P}=-P \dot{D} P
$$

is used.

The first derivative at $\hat{\mu}_{S}=0$ is

$$
\dot{G}=-2 i \operatorname{Im} \operatorname{Tr}\left[(P \dot{D} P)_{n 0} \Gamma \gamma_{5} P_{n 0}^{\dagger} \gamma_{5} \Gamma^{\dagger}\right]
$$

and the second derivative at $\hat{\mu}_{S}=0$ is obtained as

$$
\begin{aligned}
\ddot{G}= & 4 \operatorname{Re} \operatorname{Tr}\left[(P \dot{D} P \dot{D} P)_{n 0} \Gamma \gamma_{5} P_{n 0}^{\dagger} \gamma_{5} \Gamma^{\dagger}\right] \\
& -2 \operatorname{Re} \operatorname{Tr}\left[(P \ddot{D} P)_{n 0} \Gamma \gamma_{5} P_{n 0}^{\dagger} \gamma_{5} \Gamma^{\dagger}\right] \\
& -2 \operatorname{Tr}\left[(P \dot{D} P)_{n 0} \Gamma \gamma_{5}(P \dot{D} P)_{n 0}^{\dagger} \gamma_{5} \Gamma^{\dagger}\right] .
\end{aligned}
$$

Let us calculate the derivatives of the fermionic determinant $\Delta$. Using the following equations,

$$
\begin{aligned}
\frac{d}{d \hat{\mu}} \operatorname{det} D= & \operatorname{Tr}[\dot{D} P] \operatorname{det} D \\
\frac{d^{2}}{d \hat{\mu}^{2}} \operatorname{det} D= & \left\{\operatorname{Tr}[\ddot{D} P]-\operatorname{Tr}[\dot{D} P \dot{D} P]+\operatorname{Tr}[\dot{D} P]^{2}\right\} \\
& \times \operatorname{det} D,
\end{aligned}
$$

we have

$$
\begin{aligned}
& \frac{\dot{\Delta}}{\Delta}=2 \operatorname{Tr}[\dot{D} P] \\
& \frac{\ddot{\Delta}}{\Delta}=2 \operatorname{Tr}[\ddot{D} P]-2 \operatorname{Tr}[\dot{D} P \dot{D} P]+4 \operatorname{Tr}[\dot{D} P]^{2}
\end{aligned}
$$
have

Combining Eqs. (15), (A6), (A7), (A8) and (A9), we

$$
\frac{d}{d \hat{\mu}} \operatorname{Re}\left\langle H(n) H(0)^{\dagger}\right\rangle=0
$$

and 


$$
\begin{aligned}
\frac{d^{2}}{d \hat{\mu}^{2}} \operatorname{Re}\left\langle H(n) H(0)^{\dagger}\right\rangle=4 \operatorname{Re}\left\langle\operatorname{Tr}\left[(P \dot{D} P \dot{D} P)_{n 0} \Gamma \gamma_{5} P_{n 0}^{\dagger} \gamma_{5} \Gamma^{\dagger}\right]\right\rangle-2 \operatorname{Re}\left\langle\operatorname{Tr}\left[(P \ddot{D} P)_{n 0} \Gamma \gamma_{5} P_{n 0}^{\dagger} \gamma_{5} \Gamma^{\dagger}\right]\right\rangle \\
-2 \operatorname{Re}\left\langle\operatorname{Tr}\left[(P \dot{D} P)_{n 0} \Gamma \gamma_{5}(P \dot{D} P)_{n 0}^{\dagger} \gamma_{5} \Gamma^{\dagger}\right]\right\rangle+8\left\langle\operatorname{Im} \operatorname{Tr}\left[(P \dot{D} P)_{n 0} \Gamma \gamma_{5} P_{n 0}^{\dagger} \gamma_{5} \Gamma^{\dagger}\right] \operatorname{Im} \operatorname{Tr}[\dot{D} P]\right\rangle \\
+2 \operatorname{Re}\left\{\left\langle\operatorname{Tr}\left[P_{n 0} \Gamma \gamma_{5} P_{n 0}^{\dagger} \gamma_{5} \Gamma^{\dagger}\right]\left(\operatorname{Tr}[\ddot{D} P]-\operatorname{Tr}[\dot{D} P \dot{D} P]+2 \operatorname{Tr}[\dot{D} P]^{2}\right)\right\rangle\right. \\
\left.-\left\langle\operatorname{Tr}\left[P_{n 0} \Gamma \gamma_{5} P_{n 0}^{\dagger} \gamma_{5} \Gamma^{\dagger}\right]\right\rangle\left\langle\operatorname{Tr}[\ddot{D} P]-\operatorname{Tr}[\dot{D} P \dot{D} P]+2 \operatorname{Tr}[\dot{D} P]^{2}\right\rangle\right\} .
\end{aligned}
$$

\section{APPENDIX B: FORMULAE FOR THE ISOVECTOR CHEMICAL POTENTIAL RESPONSE}

Next, we consider responses to the isovector chemical potential, Eq. 17). In this case, the first derivative of $\Delta$ vanishes,

$$
\dot{\Delta}=\left.\frac{d}{d \hat{\mu}_{V}}\left(\operatorname{det} D\left[U ; \hat{\mu}_{V}\right] \operatorname{det} D\left[U ;-\hat{\mu}_{V}\right]\right)\right|_{\hat{\mu}_{V}=0}=0,
$$

and the second derivative is obtained as

$$
\frac{\ddot{\Delta}}{\Delta}=2 \operatorname{Tr}[\ddot{D} P]-2 \operatorname{Tr}[\dot{D} P \dot{D} P] .
$$

Similarly, derivatives of $G$ are calculated as

$$
\dot{G}=-2 \operatorname{Re} \operatorname{Tr}\left[(P \dot{D} P)_{n 0} \Gamma \gamma_{5} P_{n 0}^{\dagger} \gamma_{5} \Gamma^{\dagger}\right],
$$

and

$$
\begin{aligned}
\ddot{G}= & 4 \operatorname{Re} \operatorname{Tr}\left[(P \dot{D} P \dot{D} P)_{n 0} \Gamma \gamma_{5} P_{n 0}^{\dagger} \gamma_{5} \Gamma^{\dagger}\right] \\
& -2 \operatorname{Re} \operatorname{Tr}\left[(P \ddot{D} P)_{n 0} \Gamma \gamma_{5} P_{n 0}^{\dagger} \gamma_{5} \Gamma^{\dagger}\right] \\
& +2 \operatorname{Tr}\left[(P \dot{D} P)_{n 0} \Gamma \gamma_{5}(P \dot{D} P)_{n 0}^{\dagger} \gamma_{5} \Gamma^{\dagger}\right] .
\end{aligned}
$$

Resulting expressions for the first and second responses to the isovector chemical potentials are

$$
\frac{d}{d \hat{\mu}} \operatorname{Re}\left\langle H(n) H(0)^{\dagger}\right\rangle
$$

$$
=-2 \operatorname{Re} \operatorname{Tr}\left[(P \dot{D} P)_{n 0} \Gamma \gamma_{5} P_{n 0}^{\dagger} \gamma_{5} \Gamma^{\dagger}\right],
$$

and

$$
\begin{aligned}
& \frac{d^{2}}{d \hat{\mu}^{2}} \operatorname{Re}\left\langle H(n) H(0)^{\dagger}\right\rangle= \\
& 4 \operatorname{Re}\left\langle\operatorname{Tr}\left[(P \dot{D} P \dot{D} P)_{n 0} \Gamma \gamma_{5} P_{n 0}^{\dagger} \gamma_{5} \Gamma^{\dagger}\right]\right\rangle \\
& -2 \operatorname{Re}\left\langle\operatorname{Tr}\left[(P \ddot{D} P)_{n 0} \Gamma \gamma_{5} P_{n 0}^{\dagger} \gamma_{5} \Gamma^{\dagger}\right]\right\rangle \\
& +2 \operatorname{Re}\left\langle\operatorname{Tr}\left[(P \dot{D} P)_{n 0} \Gamma \gamma_{5}(P \dot{D} P)_{n 0}^{\dagger} \gamma_{5} \Gamma^{\dagger}\right]\right\rangle \\
& +2 \operatorname{Re}\left\{\left\langle\operatorname{Tr}\left[P_{n 0} \Gamma \gamma_{5} P_{n 0}^{\dagger} \gamma_{5} \Gamma^{\dagger}\right](\operatorname{Tr}[\ddot{D} P]-\operatorname{Tr}[\dot{D} P \dot{D} P])\right\rangle\right. \\
& \left.-\left\langle\operatorname{Tr}\left[P_{n 0} \Gamma \gamma_{5} P_{n 0}^{\dagger} \gamma_{5} \Gamma^{\dagger}\right]\right\rangle\langle\operatorname{Tr}[\ddot{D} P]-\operatorname{Tr}[\dot{D} P \dot{D} P]\rangle\right\} .
\end{aligned}
$$

\section{APPENDIX C: RESPONSES FOR STAGGERED FERMIONS}

For staggered fermions, the determinant factor $\Delta$ is given by Eq. (21), and this leads to

$$
\frac{\dot{\Delta}}{\Delta}=\frac{1}{2} \operatorname{Tr}[\dot{D} P]
$$

$$
\frac{\ddot{\Delta}}{\Delta}=\frac{1}{2} \operatorname{Tr}[\ddot{D} P]-\frac{1}{2} \operatorname{Tr}[\dot{D} P \dot{D} P]+\frac{1}{4} \operatorname{Tr}[\dot{D} P]^{2} .
$$

The final expressions for the second responses are

$$
\begin{aligned}
\frac{d^{2}}{d \hat{\mu}^{2}} \operatorname{Re}\left\langle H(n) H(0)^{\dagger}\right\rangle=4 \operatorname{Re}\left\langle\operatorname{Tr}\left[(P \dot{D} P \dot{D} P)_{n 0} \Gamma \gamma_{5} P_{n 0}^{\dagger} \gamma_{5} \Gamma^{\dagger}\right]\right\rangle-2 \operatorname{Re}\left\langle\operatorname{Tr}\left[(P \ddot{D} P)_{n 0} \Gamma \gamma_{5} P_{n 0}^{\dagger} \gamma_{5} \Gamma^{\dagger}\right]\right\rangle \\
-2 \operatorname{Re}\left\langle\operatorname{Tr}\left[(P \dot{D} P)_{n 0} \Gamma \gamma_{5}(P \dot{D} P)_{n 0}^{\dagger} \gamma_{5} \Gamma^{\dagger}\right]\right\rangle+2\left\langle\operatorname{Im} \operatorname{Tr}\left[(P \dot{D} P)_{n 0} \Gamma \gamma_{5} P_{n 0}^{\dagger} \gamma_{5} \Gamma^{\dagger}\right] \operatorname{Im} \operatorname{Tr}[\dot{D} P]\right\rangle \\
+\frac{1}{2} \operatorname{Re}\left\{\left\langle\operatorname{Tr}\left[P_{n 0} \Gamma \gamma_{5} P_{n 0}^{\dagger} \gamma_{5} \Gamma^{\dagger}\right]\left(\operatorname{Tr}[\ddot{D} P]-\operatorname{Tr}[\dot{D} P \dot{D} P]+\frac{1}{2} \operatorname{Tr}[\dot{D} P]^{2}\right)\right\rangle\right. \\
\left.-\left\langle\operatorname{Tr}\left[P_{n 0} \Gamma \gamma_{5} P_{n 0}^{\dagger} \gamma_{5} \Gamma^{\dagger}\right]\right\rangle\left\langle\operatorname{Tr}[\ddot{D} P]-\operatorname{Tr}[\dot{D} P \dot{D} P]+\frac{1}{2} \operatorname{Tr}[\dot{D} P]^{2}\right\rangle\right\}
\end{aligned}
$$


for the isoscalar chemical potential, and

$$
\begin{aligned}
\frac{d^{2}}{d \hat{\mu}^{2}} \operatorname{Re}\left\langle H(n) H(0)^{\dagger}\right\rangle= & 4 \operatorname{Re}\left\langle\operatorname{Tr}\left[(P \dot{D} P \dot{D} P)_{n 0} \Gamma \gamma_{5} P_{n 0}^{\dagger} \gamma_{5} \Gamma^{\dagger}\right]\right\rangle-2 \operatorname{Re}\left\langle\operatorname{Tr}\left[(P \ddot{D} P)_{n 0} \Gamma \gamma_{5} P_{n 0}^{\dagger} \gamma_{5} \Gamma^{\dagger}\right]\right\rangle \\
+ & 2 \operatorname{Re}\left\langle\operatorname{Tr}\left[(P \dot{D} P)_{n 0} \Gamma \gamma_{5}(P \dot{D} P)_{n 0}^{\dagger} \gamma_{5} \Gamma^{\dagger}\right]\right\rangle \\
+ & \frac{1}{2} \operatorname{Re}\left\{\left\langle\operatorname{Tr}\left[P_{n 0} \Gamma \gamma_{5} P_{n 0}^{\dagger} \gamma_{5} \Gamma^{\dagger}\right](\operatorname{Tr}[\ddot{D} P]-\operatorname{Tr}[\dot{D} P \dot{D} P])\right\rangle\right. \\
& \left.-\left\langle\operatorname{Tr}\left[P_{n 0} \Gamma \gamma_{5} P_{n 0}^{\dagger} \gamma_{5} \Gamma^{\dagger}\right]\right\rangle\langle\operatorname{Tr}[\ddot{D} P]-\operatorname{Tr}[\dot{D} P \dot{D} P]\rangle\right\}
\end{aligned}
$$

for the isovector chemical potential.

[1] M. A. Stephanov, Phys. Rev. Lett. 76, 4472 (1996).

[2] T. Hatsuda and S. H. Lee, Phys. Rev. C 46, 34 (1992).

[3] A. Hayashigaki, Phys. Lett. B 487, 96 (2000).

[4] CERES Collaboration, G. Agakishiev et al., Nucl. Phys. A638, 159 (1998).

[5] NA50 collaboration, M. C. Abreu et al., Phys. Lett. B 477, 28 (2000); See also H. Satz, Nucl. Phys. B (Proc.Suppl.) 94, 204 (2001).

[6] R. Rapp and C. Gale, Phys. Rev. C 60, 024903 (1999); J. Stachel, Nucl. Phys. A 654, 119C (1999); R. Rapp and J. Wambach, nucl-th/0001014.

[7] I. M. Barbour, S. E. Morrison, E. G. Klepfish, J. B. Kogut and M. Lombardo, Nucl. Phys. B (Proc.Suppl.) 60A, 220 (1998).

[8] F. Karsch, Nucl. Phys. B (Proc. Suppl.) 83-84, 14 (2000); O. Kaczmarek, J. Engels, F. Karsch, and E. Laermann, Nucl. Phys. B (Proc.Suppl.) 83-84, 369 (2000); G. Aarts, F. Karsch, O. Kaczmarek and I.-O. Stamatescu, "First order correction to quenched QCD at non-zero chemical potential", contribution to Lattice 2001.

[9] A. Hart, M. Laine, and O. Philipsen, Phys. Lett. B 505, 141 (2001).
[10] S. Gottlieb et al., Phys. Rev. D 55, 6852 (1997).

[11] QCD-TARO Collaboration, Ph. de Forcrand et al., Nucl. Phys. B (Proc.Suppl.) 63 A-C, 460 (1998); Nucl. Phys. B (Proc. Suppl.) 73, 477 (1999).

[12] S. Gottlieb, W. Liu, D. Toussaint, R. L. Renken and R. L. Sugar, Phys. Rev. D 38, 2888 (1988).

[13] D. T. Son and M. A. Stephanov, Phys. Rev. Lett. 86, 592 (2001).

[14] M. Fukugita, H. Mino, M. Okawa and A. Ukawa, Phys. Rev. D 42, 2936 (1990).

[15] S. Gottlieb, W. Liu, D. Toussaint, R. L. Renken and R. L. Sugar, Phys. Rev. D 35, 3972 (1987).

[16] S. Dong and K. Liu, Phys. Lett. B 328, 130 (1994).

[17] S. Gottlieb et al., Phys. Rev. D 47, 3619 (1993).

[18] M. Fukugita et al., Phys. Rev. Lett. 68, 761 (1992); Phys. Rev. D 47, 4739 (1992); S. Aoki et al., ibid. D 50, 486 (1994).

[19] C. Bernard et al., Phys. Rev. D 48, 4419 (1993).

[20] CP-PACS Collaboration, A. Ali Khan et al. heplat/0103028.

[21] O. Miyamura and S. Choe, hep-ph/0105198 